\title{
A satisfação dos editores das revistas do Portal de Periódicos da FURG em relação ao Sistema Eletrônico de Editoração de Revistas (SEER)
}

\author{
Tatiane Priscila Pinto Corrêa \\ Universidade Federal de Santa Catarina - Brasil \\ Angélica Conceição Dias Miranda \\ Universidade Federal de Santa Catarina - Brasil
}

ESTUDOS / CASES

\begin{abstract}
Resumo
O presente artigo tem por objetivo divulgar os resultados obtidos através de uma pesquisa realizada com os editores das revistas do Portal de Periódicos Científicos, da Universidade Federal do Rio Grande (FURG), a respeito da satisfação em relação ao Sistema Eletrônico de Editoração de Revistas (SEER). A pesquisa tem por natureza quanti-qualitativa e o universo de pesquisa foi os editores das dez revistas que compõem o Portal. O instrumento de coleta de dados escolhido foi um questionário composto por dez perguntas fechadas e uma pergunta aberta. Para realização da investigação, o questionário foi enviado para o e-mail de cada editor. Os resultados demonstraram que os pesquisados encontram-se satisfeitos com o processo de editoração eletrônica. Além dos resultados quantitativos, alguns editores fizeram comentários e sugestões importantes que visam incentivar a ampliação da qualidade e visibilidade das revistas eletrônicas da instituição.
\end{abstract}

\section{Palavras-chave}

Estudo do usuário ; Comunicação científica ; Sistema de Editoração Eletrônica de Revistas - SEER ; Editores de revistas científicas ; Revistas científicas ; Portal de Periódicos da FURG

\section{The editors' satisfactions of the Portal of Scientific Journals of FURG with respect to the Sistema Eletrônico de Editoração de Revistas (SEER)}

\begin{abstract}
This article aims to disseminate the results obtained from a survey with the editors of the Portal of Scientific Journals, of the Universidade Federal do Rio Grande (FURG), about the satisfaction with the Sistema Eletrônico de Editoração de Revistas (SEER). The research is quantitative and qualitative nature and the universe of research has been the editors of ten journals. The data collection instrument was a questionnaire consisting of ten closed questions and one open question. To carry out the research, the questionnaire was mailed to the e-mail each editor. The results showed that the respondents were satisfied with the process of electronic publishing. In addition to the quantitative results, some publishers have made important comments and suggestions aimed at encouraging the expansion of quality and visibility of electronic journals of the institution.
\end{abstract}

Keywords

User study; Scientific communication ; Sistema de Editoração Eletrônica de Revistas - SEER ; Editors of scientific journals ; Portal de Periódicos da FURG 


\section{Introdução}

As importâncias da comunicação científica para o crescimento da ciência e progresso da sociedade na contemporaneidade aliada aos avanços em tecnologia viabilizaram o surgimento de ferramentas que objetivam facilitar e otimizar diversas atividades, que até antes eram realizadas em meio tradicional, e agora podem ser desempenhadas em meio eletrônico, como por exemplo, a editoração de revistas científicas em formato on-line. $O$ Open Journal System (OJS), desenvolvido pelo Public Knowledge Project (PKP) é um software livre de gestão de periódicos eletrônicos, que permite realizar todos os procedimentos de editoração - desde submissão de papers, passando pelo processo de avaliação por pares e às cegas, até o momento da publicação. O Instituto Brasileiro de Informação em Ciência e Tecnologia (IBICT) foi a instituição brasileira responsável em traduzir o OJS para a língua portuguesa, originando o Sistema Eletrônico de Editoração de Revistas (SEER).

O presente artigo tem por objetivo divulgar os resultados obtidos através de uma pesquisa realizada com os editores do Portal de Periódicos da FURG.

A pesquisa teve por finalidade conhecer a satisfação dos editores quanto à ferramenta SEER, bem como quanto os serviços prestados pela Equipe SEER/FURG, NTI/FURG (antigo CPD) e infra-estrutura disponibilizada pela universidade para o desenvolvimento das revistas eletrônicas. A justificativa para a realização deste estudo foi identificada a partir da prática de editoração eletrônica em conjunto com os editores das revistas, onde sentiu-se a necessidade de saber se os mesmos estão satisfeitos com o sistema. A pesquisa tem por características: natureza quantitativa, do tipo descritiva, de finalidade básica e temporalidade transversal. O instrumento de coleta de dados adotado foi um questionário em formato MS Word, contendo dez perguntas fechadas e uma pergunta aberta, que foi enviado para os endereços eletrônicos dos pesquisados.

\section{Comunicação científica: um breve resumo}

O início dos anos 80 marcou profundamente as estruturas tecnológicas a partir da produção em massa dos microcomputadores. Os equipamentos que antes estavam restritos aos ambientes corporativos e militares, agora estão mais mais acessíveis e pessoais, conforme ilustra Meadows (1999):

Segundo Merlo Vega (2000):

Os computadores eletrônicos eram, naturalmente, equipamentos caros até os microcomputadores se tornarem comuns na década de 1980. Grande parte das atividades iniciais que envolviam o processamento de informação com computadores contou com o financiamento de fontes militares (p. 33).

Nota-se em geral, que o fluxo de informacional aumentou em consonância com os avanços em tecnologia. Em decorrência do advento das Novas Tecnologias da Informação e Comunicação (NTIC's), surgiram de novas ferramentas, resultando em inovadoras alternativas de otimizar o tempo das pessoas em suas inúmeras tarefas cotidianas.

Os anos 80 viram o prenúncio do horizonte contemporâneo da multimídia. A informática perdeu, pouco a pouco, seu status de técnica e de setor industrial particular para começar a fundir-se com as telecomunicações, a editoração, o cinema e a televisão. A digitalização penetrou primeiro na produção e na gravação de músicas, mas os microprocessadores e as memórias digitais tendiam a tornar-se a infra-estrutura de produção de todo o domínio da comunicação. Novas formas de mensagens "interativas" apareceram: este decênio viu a invasão dos videogames, o triunfo da informática "amigável" (interfaces gráficas e interações sensório-motoras) e o surgimento dos hiperdocumentos (hipertextos, CD-ROM) (Levy, 1999, p. 32).

A informação é o principal agente promotor do desenvolvimento social, científico e tecnológico. Portanto, é necessário que os resultados da produção intelectual sejam divulgados para que todos tenham acesso à essas informações, que sejam conhecidas, verificadas e continuadas em estudos futuros. Também vale ressaltar, que a divulgação desses trabalhos é a base para o crescimento de um país frente as potencias mundiais. 
A produção científica como fruto da construção do conhecimento individual ou coletivo propicia a potencial visibilidade evolutiva dos delineamentos teóricos gerados pelos pesquisadores bem como contribui para o enriquecimento de novos saberes. No entanto, como uma atividade eminentemente social, precisa ser comunicada para que alcance o seu reconhecimento entre seus pares e também motive outras descobertas (Grants, Oliveira, Philippi, 2011, p. 5).

A comunicação científica, como é chamada a divulgação de trabalhos científicos em eventos e publicações científicas, é definida por Marconi e Lakatos (2009) como:

Comunicação científica é a informação apresentada em congressos, simpósios, semanas, reuniões, academias, sociedades científicas etc., onde se expõem os resultados de uma pesquisa original, inédita, criativa, a ser publicada posteriormente em anais ou revistas (p. 79).

A importância da publicação da produção intelectual em periódicos científicos está cada vez mais em evidência na comunidade, uma vez que esses textos são encaminhados e revisados por especialistas da área temática, atribuindo aos periódicos a confiabilidade das informações neles contidas. Kuramoto (2007) ilustra a importância dos periódicos científicos para a disseminação dos resultados de pesquisas científicas:

O periódico científico tem sido considerado, para as ciências exatas e naturais, assim como para parte significativa das ciências humanas e sociais e para parcela menor das artes e humanidades, o veículo mais importante de comunicação da pesquisa. Representa, assim, um dos veículos que compõem o produto final, formal, consolidado da disseminação de resultados de pesquisas realizadas por estudiosos de todo o mundo (p. 3).

O grande fluxo informacional e o crescimento de novas revistas científicas, ambos impulsionados pelo avanço tecnológico, possibilitou o surgimento de novas ferramentas que permitem agilizar os processos de editoração tradicional, que antes eram sinônimos de morosidade - em relação ao tempo de envio, transporte e entrega dos papers para os editores e avaliadores, envio de pareceres através de agências de correspondências etc.

Na década de 1980, o desenvolvimento da tecnologia da informação alcançara a etapa em que podia começar a competir com a impressão em papel como meio universal para difundir informações científicas. Nos últimos anos, portanto, passou a ser razoável examinar a possibilidade de se transferirem informações científicas do meio impresso para o meio eletrônico (Meadows, 1999, p. 35).

Com todas estas inovações, o reconhecimento deste novo formato, o on-line, indica que o periódico científico seja o veículo mais eficiente para a disseminação da comunicação científica, agora em tempo real, facilitando a troca de informações entre os pares e a evolução da ciência, do conhecimento[...] (Fachin, Hillesheim, 2006, p. 38).

\section{Uma nova alternativa: tecnologia $x$ comunicação científica}

A tecnologia veio ao encontro da comunicação científica e como fruto dessa união, surgiu uma proposta interessante: a partir do suporte de computadores e da Internet, transportar todo o processo de editoração tradicional para o meio eletrônico, onde todos os passos percorridos serão realizados através de um sistema on-line, que possibilitará o gerenciamento de todo o processo de editoração, bem como da própria revista e dos atores que contribuem para o sucesso do trabalho editorial. Como resultado desta inovação, surge então, as revistas eletrônicas. Meadows (1999) comenta outros aspectos importantes que uma revista eletrônica pode conter, além de suas semelhanças com a revista impressa:

Uma revista eletrônica pode adotar métodos semelhantes ao do periódico impresso ao procurar resolver muitos desses problemas, mas conta ainda com o potencial de ser mais flexível. Por exemplo, o leiaute clássico da revista impressa pode ser reproduzido facilmente na tela (levando em conta os diferentes formatos e possibilidades da página impressa e da tela), porém o computador oferece recursos adicionais[...]. No caso da revista eletrônica, ela pode ser organizada de tal forma que os outros materiais citados no texto de um artigo possam ser imediatamente trazidos à tela com o apertar de um botão (p. 36). 
Um dos exemplos de sistemas de editoração eletrônica é o Open Journal Systems (OJS). Ele foi desenvolvido pelo Public Knowledge Project (PKP), da Universidade British Columbia, no Canadá. Esse sistema permite a gestão de todo o processo editorial de uma revista científica em meio eletrônico. Além disso, ele é uma ferramenta gratuita, que permite avaliação por pares e às cegas, que garante confiabilidade e veracidade no momento da avaliação e publicação os artigos submetidos à revista. Atualmente, o Open Journal Systems, encontra-se instalado em 9.000 revistas em todo mundo, segundo a última estatística publicada no site do PKP ${ }^{1}$.

A questão fundamental é quanta informação científica entra em circulação a cada ano. Uma resposta abrangente exige um estudo de todos os veículos de comunicação. Nas últimas décadas, cada vez mais os pesquisadores participam do processamento de informações por computador, ao ponto de o armazenamento e a comunicação de informações científicas por meios eletrônicos ser hoje coisa corriqueira. Em geral, se supõe que os fatos atuais prenunciam uma transferência em grande escala da comunicação científica dos meios impressos para os meios eletrônicos. Se assim for, essa será uma importante revolução, pois a comunidade científica, desde a infância, depende da impresa (Meadows, 1999, p. 32).

O Instituto Brasileiro de Informação em Ciência e Tecnologia (IBICT) foi a instituição brasileira responsável pela customização e tradução do OJS para a língua portuguesa, originando o Sistema Eletrônico de Editoração de Revistas (SEER). Além de disponibilizar a ferramenta, suporte e treinamento, o IBICT se preocupa em cadastrar todas as revistas científicas brasileiras que fazem o uso do SEER, como forma de obter um controle estatístico e promover maior divulgação do acesso livre à informação. Segundo a última estatística divulgada no Portal do IBICT existem 598 revistas cadastradas em seu banco de dados².

\section{O Portal de Periódicos da FURG}

O Portal de Periódicos da FURG conta com o elenco de 11 revistas no total, são elas: Ambiente \& Educação, Atlântica (Rio Grande), Biblos, Juris, Momento, Revista Didática Sistêmica, Revista Brasileira de Educação Ambiental (REVBEA), Revista Eletrônica do Mestrado de Educação Ambiental (REMEA), Sinergia, Vetor e Vittalle.

A utilização do Sistema Eletrônico de Editoração de Revistas (SEER) nos processos editoriais facilita, agiliza e reduz os custos - em relação ao processo de editoração tradicional, através do suporte papel - no momento de submissão e avaliação dos artigos das revistas científicas.

A Equipe do SEER/FURG é composta por 11 bolsistas (graduandos do curso de Biblioteconomia), bolsistas voluntários, coordenadora do Portal e colaboradores. Os serviços prestados são: assistência aos editores, inserção e padronização de metadados em planilhas no Google Docs, indexação, marketing, treinamento, palestras, promoção de encontros e reuniões para debates entre outras ações que visam buscar a ampliação da qualidade e visibilidade das revistas da universidade.

Conhecer a satisfação dos editores responsáveis pelas revistas é um fator importante para aprimorar, cade vez mais, o trabalho realizado pela equipe. Meadows (1999) explica a importância do papel dos editores no controle de qualidade dos periódicos científicos:

Deve haver, portanto, alguma forma de controle de qualidade, aprovada pela comunidade, que possa ser aplicada ao material novo. Esse controle é exercido, em primeira instância, pelos editores, que atuam como gatekeepers. Assumem a responsabilidade de decidir se uma colaboração chegará a ser publicada e, em caso afirmativo, como será apresentada (p. 180).

\footnotetext{
${ }^{1}$ Estatística realizada em abril de 2011, pela PKP. Recuperado em 25 junho, 2011, de http://pkp.sfu.ca/ojs-usernumbers.

${ }^{2}$ Estatística realizada pela instituição. Recuperado em 06 maio, 2011, de

http://seer.ibict.br/index.php?option=com content\&task=view\&id=395\&ltemid=120.
} 


\section{Análise dos resultados}

O instrumento de coleta de dados adotado foi questionário composto por dez perguntas fechadas e uma pergunta aberta - ao final do questionário para opiniões, sugestões ou críticas. Os questionários foram enviados para os emails de 10 revistas do Portal de Periódicos da FURG ${ }^{3}$. Dos 10 pesquisados contatados, 08 responderam os questionários em tempo hábil à tabulação dos dados. Ressaltamos que o instrumento foi reenviado para os editores que ainda não haviam respondido, porém não foi possível obter os questionários respondido por eles.

Perguntamos "Há quanto tempo você desempenha a função de editor de revista(s) científica(s)?". Como resultado obtivemos: $37 \%$ responderam de 6 meses -1 ano; $25 \%$ menos de 6 meses; $25 \%$ acima de 4,5 anos; $13 \%$ de 3,5 anos - 4 anos, conforme ilustra o Gráf. 1:

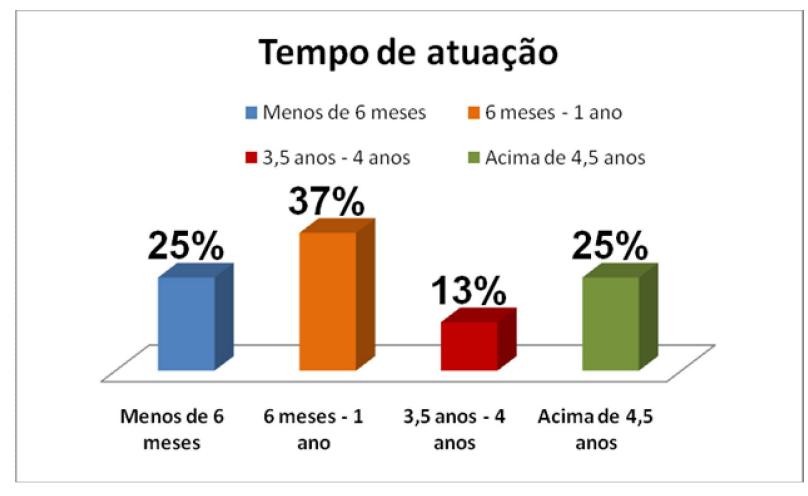

Gráfico 1 - Tempo de atuação dos editores respondentes na função editorial. Fonte: Autora

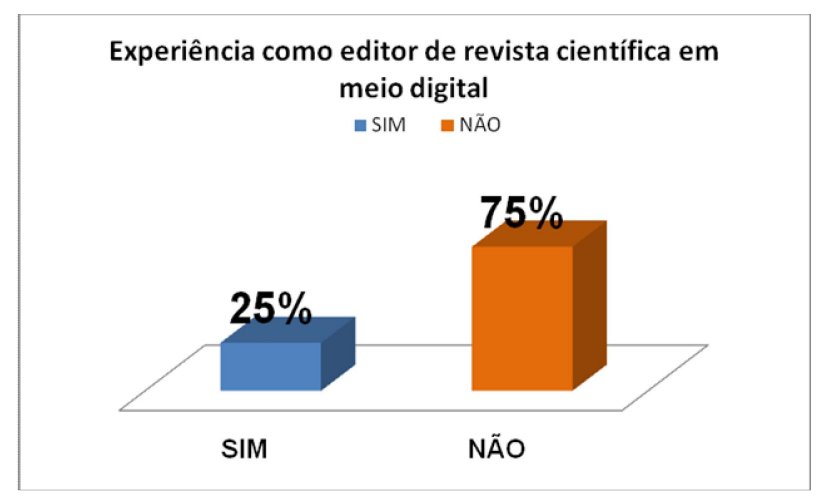

Gráfico 2 - Experiência como editor de revista científica em meio digital.

Fonte: Autora

Os números demonstraram que parte dos pesquisados desempenham a pouco tempo a função de editor de revistas científicas, enquanto houve um equilíbrio entre editores que possuem menos de 6 meses e dos que possuem mais de 4,5 anos de atuação.

$\mathrm{Na}$ questão seguinte, foi perguntado aos pesquisados se já possuiam alguma experiência anterior como editor de revistas científicas em meio eletrônico. Como resposta, $75 \%$ dos editores não tiveram nenhuma função como editor de revistas eletrônicas anteriormente, enquanto $25 \%$ afirmaram que já atuaram (Gráf. 2). Com base nestes número percebemos que mais da metade dos editores do Portal estão vivenciando suas primeiras experiências como editores de revista científica em meio eletrônico.

Outra questão abordou o tempo que eles conheciam o Sistema Eletrônico de Editoração de Revistas (SEER), tivemos como resultados que $37 \%$ afirmaram que conhecem o sistema entre o período de 6 meses -1 ano, $25 \%$ de 3,5 anos -4 anos, $13 \%$ de 2,5 anos -3 anos, $13 \%$ de 4,5 anos acima, $12 \%$ menos de 6 meses (Gráf. 3). No momento da tabulação dos dados, sentiu-se a necessidade de complementar essa questão com outra que especificasse como o editor conheceu o sistema. Pensamos que através dessa pergunta, pudéssemos delimitar qual experiência oportunizou os editores conhecerem o sistema (se foi através de treinamento ou já havia submetido artigos através do sistema etc.).

${ }^{3}$ Em 2010, ano de realização da pesquisa, o Portal de Periódicos da FURG contava com 10 revistas. 


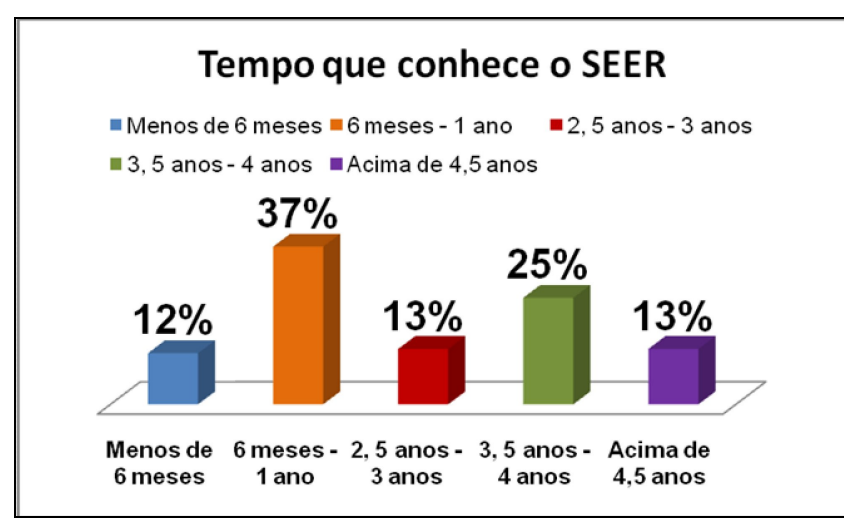

Gráf. 3 - Tempo que os editores respondentes conhecem o SEER.

Fonte: Autora

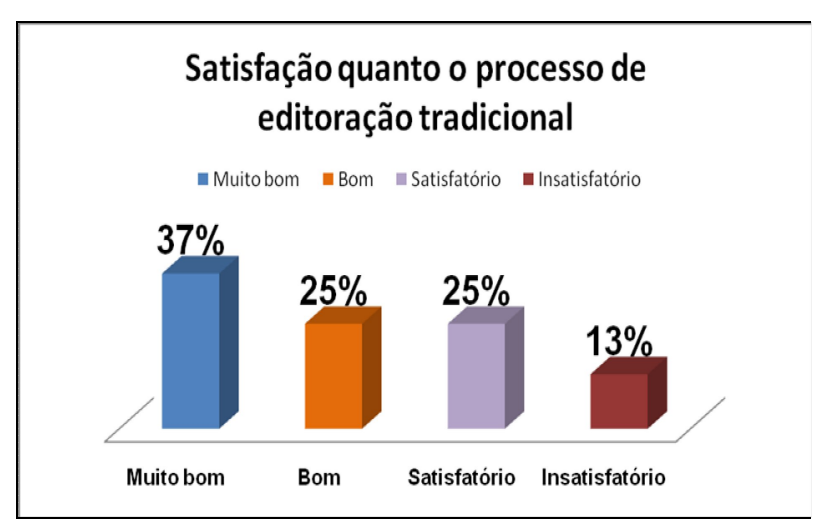

Gráf. 5 - Satisfação dos editores quanto o processo de editoração tradicional (via correios - suporte papel).

Fonte: Autora

Na questão "Qual sua satisfação quanto o processo editorial tradicional?", responderam que: 37\% consideraram muito bom, $25 \%$ bom, outros 25\% encontram-se satisfeitos, enquanto $13 \%$ encontram-se insatisfeitos (Gráf. 5). Segundo os números, os editores avaliam o processo editorial tradicional positivamente, já que a maior parte das respostas oscilaram entre o muito bom e bom, e outros satisfatoriamente.

A questão seguinte abordou a principal pergunta da pesquisa: "Qual é a sua satisfação quanto processo editorial através do Sistema Eletrônico de Editoração de Revistas - SEER?”, como resultados obtivemos: 62\% consideraram muito bom, $25 \%$ afirmaram que é bom, enquanto $13 \%$ não soube responder ou não respondeu (Gráf. 6). 0 percentual de $13 \%$ se justifica pelo fato de um dos pesquisados assinalar no questionário que ele não poderia responder a questão, visto que sua revista é nova no Portal e ainda não tive experiência para avaliar o sistema. Esses resultados demonstraram a boa aceitação do SEER entre os editores das revistas científicas eletrônicas da FURG.

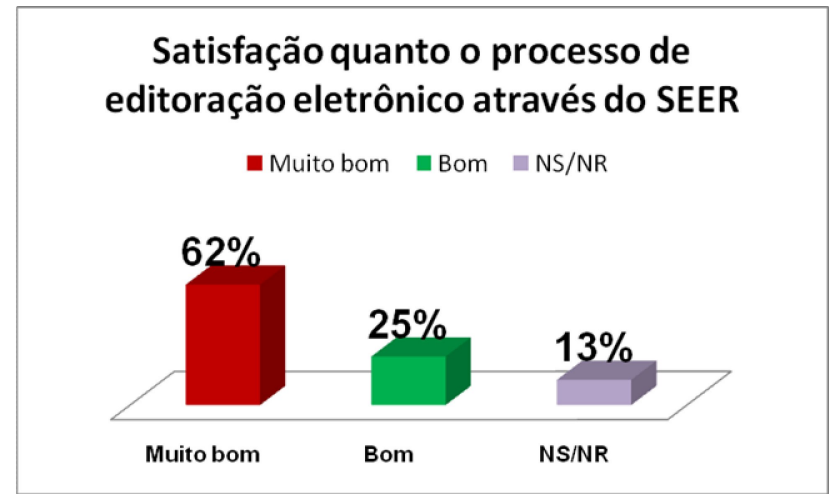

Gráf. 6 - Satisfação dos editores quanto o processo editorial através do SEER.

Fonte: Autora

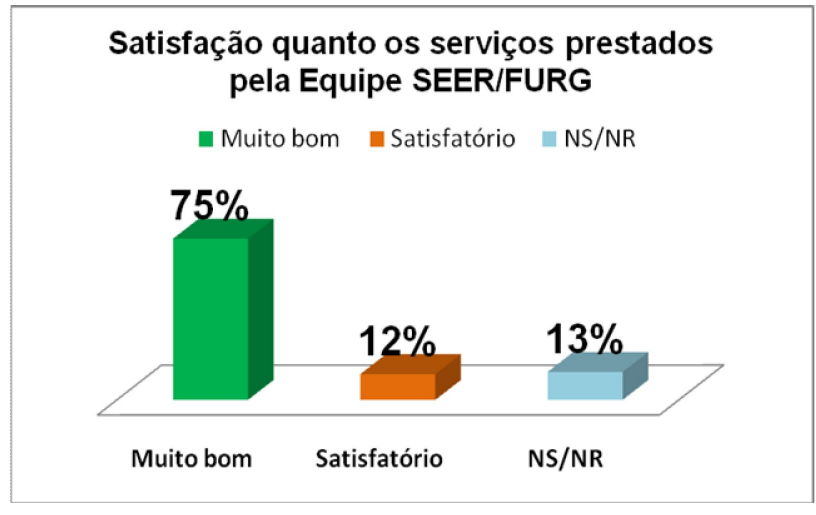

Gráf. 7 - Satisfação dos editores quanto os serviços prestados pela Equipe do SEER/FURG.

Fonte: Autora 
Perguntamos a satisfação dos editores quanto os serviços prestados pela Equipe do SEER da FURG: 75\% afirmaram que os serviços são considerados muito bom, 12\% consideram satisfatório e $13 \%$ não souberam responder ou não responderam (Gráf. 7). Mais uma vez, o percentual de $13 \%$ se repetiu pelo fato de um dos editores assinalar que sua revista é nova no sistema e com isso ainda não teve tempo suficiente para avaliar a equipe.

Também foi perguntado a satisfação dos pesquisados quanto o apoio da Universidade, sobre serviços oferecidos para os editores através do Núcleo de Tecnologia da Informação (anteriormente chamado de CPD). Os resultados demonstraram que $25 \%$ consideram muito bom, enquanto $25 \%$ insatisfatório, $12 \%$ bom, $12 \%$ regular, $13 \%$ satisfatório, outros 13\% não souberam responder ou não responderam (Gráf. 8). As opiniões dos editores divergiram nesta questão, já que metade avalia como muito bom, bom e satisfatório os serviços oferecidos pelo Núcleo e outra metade considera regular ou insatisfatório os serviços.

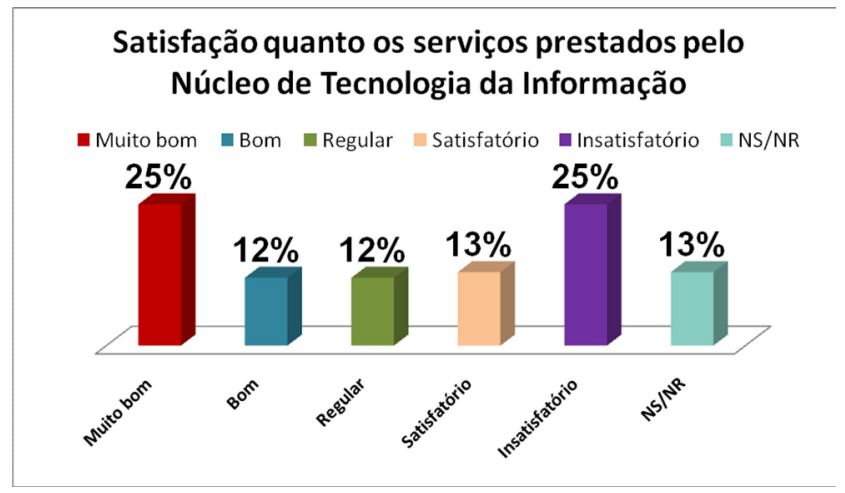

Graf. 8 - Satisfação dos editores quantos os serviços oferecidos pelo Núcleo de Tecnologia da Informação. Fonte: Autora

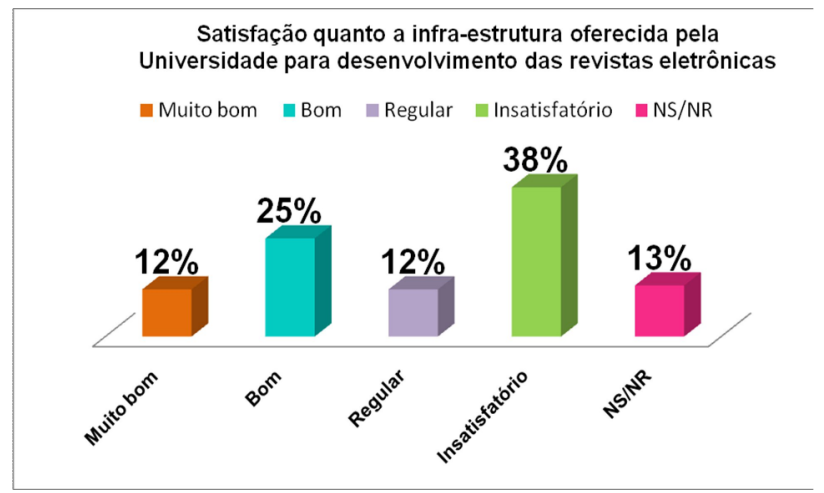

Graf. 9 - Satisfação dos editores quanto a infraestrutura oferecida pela Universidade para desenvolvimento das revistas eletrônicas. Fonte: Autora

Os resultados da questão, "Qual sua satisfação quanto o apoio da Universidade, sobre a infra-estrutura (sala, recursos humanos e tecnológicos) oferecida para desenvolvimento das Revistas no suporte online?', demonstraram que $38 \%$ dos editores consideram insatisfatório, 25\% bom, 13\% não souberam responder ou não responderam, 12\% regular e outros $12 \%$ muito bom (Gráf. 9). Mais uma vez, os resultados não foram unificados, ainda assim, maior parte dos editores respondentes encontram-se insatisfeitos quanto a infra-estrutura oferecida pela Universidade.

A última pergunta do questionário, revelou alguns comentários importantes que serão mostrados a seguir. Um dos editores, revelou sua opinião sobre a relevância da pesquisa para a maximização do apoio da Universidade para com as revistas eletrônicas da instituição: "[a] pesquisa é importante e pode contribuir para melhorar a utilização do sistema SEER na FURG, pois é preciso melhorar o apoio da instituição no desenvolvimento das revistas do SEER", os comentários dos editores é quase unanime quanto esse quesito, e também podemos confirmar esse comentário através do demonstrativo dos gráficos da pesquisa, que a instituição precisa aumentar o incentivo no desenvolvimento das revistas da Universidade.

Outro comentário aborda a questão do número de bolsistas disponíveis para cada revista: "Ainda há necessidade de muitas melhorias, apesar do interesse da equipe do SEER e dos editores. Cada revista precisaria de um bolsista para acompanhar sistematicamente as submissões, os pareceres enviados pelos avaliadores e para providenciar o encaminhamento dos mesmos aos autores. O processo é lento e ainda complicado para alguns autores. Muitos pareceres são enviados via e-mail, pois os avaliadores encontram dificuldades no sistema. Ocasionalmente conseguimos um encontro com o/a bolsista para dar andamento aos trabalhos. Penso que somente quando cada revista tiver seu bolsista poderemos dar um melhor atendimento aos autores e avaliadores contribuindo para a 
melhoria da qualidade da revista. Seria importante que cada editor com seu bolsista pudesse ter uma sala com todo o material e computadores disponíveis[...]".

Também foi comentada a falta de suporte de um profissional especializado na área da Tecnologia da Informação para realizar mudanças no sistema: "[...] único e maior problema quanto ao sistema é a falta de alguém de suporte da informática que possa prover mudanças, mesmo que simples, no sistemas. Estamos nas "mãos" do sistema. Mesmo que ele seja excelente, eventualmente, é preciso fazer algumas alterações e aí não temos suporte técnico nenhum".

A questão da qualificação dos periódicos da instituição foi levantada por outro pesquisado: "A universidade necessita adequar-se à uma nova realidade: dos periódicos eletrônicos. É preciso trabalhar pela qualificação dos mesmos, não há como evitar".

\section{Considerações finais}

Concluímos que os avanços em tecnologia trouxeram inúmeros benefícios para a sociedade, não somente com o objetivo de tornar a vida das pessoas mais fácil e dinâmica, mas também pela redução de custos e tempo que essas gastariam ao realizar suas tarefas de maneira tradicional.

Conforme comprovam as estatísticas do Instituto Brasileiro de Informação em Ciência e Tecnologia (IBICT), o número de revistas científicas online que utilizam o SEER está crescendo cada vez mais, portanto, podemos concluir que, a partir desses resultados e também pela amostragem abordada por esta pesquisa, o sistema está satisfazendo as expectativas da comunidade científica.

Através desta pesquisa, podemos perceber que os editores pesquisados estão satisfeitos com o processo editorial eletrônico, bem como com os serviços oferecidos pela Equipe SEER/FURG. Esse resultado demonstra que a instituição, deve mais do que nunca, continuar a acreditar na potencialidade do Portal, da revistas e seus editores, bem como equipe, além de investir mais no aperfeiçoamento de pessoal e divulgação do sistema na Universidade.

Os comentários dos editores, ao final do questionário, revelaram vários aspectos importantes para o crescimento das revistas e do Portal. Ressaltamos que dentre os comentários feitos, um dos mais importantes a ser providenciado é o aumento de pessoal especializado (técnicos em TI e bolsistas) e melhorias no desenvolvimento e qualificação das revistas do Portal.

A ampliação da qualidade e visibilidade das revistas do Portal de Periódicos da Universidade Federal do Rio Grande é substancial para que a instituição ostente o prestígio e relevância de seus periódicos para a comunidade científica. Portanto, não se deve medir esforços para tentar alcançar, cada vez mais, o maior nível de qualidade das publicações eletrônicas da Universidade.

\section{Referências}

BAPTISTA, A. A., Costa, S. M. de S., Kuramoto, H., Rodrigues, E. (2007).Comunicação científica: o papel da Open Archives Initiative no contexto do Acesso Livre. Encontros Bibli (número especial), 1-17. Recuperado em 24 junho, 2011, de http://repositorium.solum.uminho.pt/handle/1822/8727

FACHIN, G. R. B., HILLESHEIM, A. I. de A. (2006). Periódico científico: padronização e organização. Florianópolis: UFSC.

GRANTS, A. F. L., OLIVEIRA, A. P., PHILIPPi, T. B. (2011). Sistema eletrônico de editoração de revista (SEER): processo editorial. Florianópolis: UFSC/Biblioteca Central. Recuperado em 6 maio, 2011, de http://www.bu.ufsc.br/design/e-book-seer-ufsc-unisul-1.pdf

LÈVY, P. (1999). Cibercultura. São Paulo: Ed. 34.

MARCONI, M. de A., LAKATOS, E. M. (2009). Metodologia do trabalho científico. São Paulo: Atlas.

MEADOWS, A. J. (1999). A comunicação científica. Brasília: Briquet de Lemos/Livros. 


\section{Dados das autoras}

Tatiane Priscila Pinto Corrêa

Graduanda $7^{\circ}$ período do curso de Biblioteconomia, pela Universidade Federal do Rio Grande (FURG). Atualmente é estagiária da Divisão de Bibliotecas, da Secretaria Municipal de Educação e Cultura (SMEC), da Prefeitura Municipal do Rio Grande. Assistente de Editor da Revista Ambiente \& Educação - Revista de Educação Ambiental, do Programa de Pós-Graduação em Educação Ambiental, da Universidade Federal do Rio Grande.

tppcorrea@gmail.com

Angélica Conceição Dias Miranda

Professora no Instituto de Ciências Humanas e da Informação (ICHI), Universidade Federal do Rio Grande (FURG). Doutora em Engenharia e Gestão do Conhecimento pela Universidade Federal de Santa Catarina (UFSC).

angelicacdm@gmail.com

Recebido - Received: 2011-07-04

Aceito - Accepted: 2011-09-30

\section{(cc) EY-NC-ND}

This work is licensed under a Creative Commons Attribution-Noncommercial-No Derivative Works 3.0 United States License.

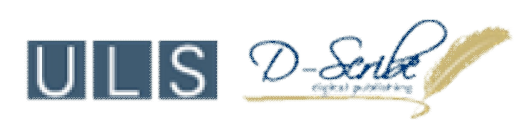

This journal is published by the University Library System of the University of Pittsburgh as part of its $\underline{D-S c r i b e}$ Digital Publishing Program and is cosponsored by the University of Pittsburgh Press. 